\title{
Total Synthesis of Tiacumicin B: Study of the Challenging $\beta$-Selective Glycosylations
}

Cédric Tresse, ${ }^{[\mathrm{a}]}$ Marc François-Heude, ${ }^{[\mathrm{a}]}$ Vincent Servajean, ${ }^{[\mathrm{a}]}$ Rubal Ravinder, ${ }^{\mathrm{a}]}$ Clémence Lesieur, ${ }^{[\mathrm{a}]}$ Lucie Geiben, ${ }^{[\mathrm{a}]}$ Louis JeanneJulien, ${ }^{[\mathrm{b}]}$ Vincent Steinmetz, ${ }^{[\mathrm{a}]}$ Pascal Retailleau, ${ }^{[\mathrm{a}]}$ Emmanuel Roulland*[b], Jean-Marie Beau, ${ }^{[\mathrm{a}][\mathrm{c}]}$ Stéphanie Norsikian. ${ }^{*[\mathrm{a}]}$

[a] Université Paris-Saclay, CNRS, Institut de Chimie des Substances Naturelles, UPR 2301, 91198, Gif-sur-Yvette (France). [b] C-Tac, CitCom, UMR 8038, CNRS-Université de Paris, Faculté de Pharmacie, 4, avenue de l'Observatoire,75006, Paris (France). [c] Institut de Chimie Moléculaire et des Matériaux d'Orsay (ICMMO), UMR 8182, Univ. Paris-Sud and CNRS, Université Paris-Saclay, F-91405

Orsay, France

KEYWORDS: Antibiotics, Natural Products, Total Synthesis, 1,2-Cis-Glycosylation, Glycochemistry, Catalysis, Palladium Chemistry.

\begin{abstract}
We report here a full account of the total synthesis of tiacumicin B (Tcn-B), a natural glycosylated macrolide with remarkable antibiotic properties. Our strategy is based on our experience with the synthesis of the tiacumicin B aglycone and on unique 1,2-cisglycosylation steps. It features the conclusive use of sulfoxide anomeric leaving-groups in combination with a remote 3-O-picoloyl group on the donors allowing highly $\beta$-selective rhamnosylation and noviosylation that rely on H-bond-mediated Aglycone Delivery (HAD). The rhamnosylated C1-C3 fragment was anchored to the C4-C19 aglycone fragment by a Suzuki-Miyaura cross-coupling. Ring-size selective Shiina macrolactonization provided a semi-glycosylated aglycone that was engaged directly in the noviolysation step with a virtually total $\beta$-selectivity. Finally, a novel deprotection method was devised for the removal of a 2-naphthylmethylidene (Nap) ether on a phenol and efficient removal of all the protecting groups provided synthetic tiacumicin B.
\end{abstract}

Nowadays antibiotic resistance is one of the most serious threats to global health, food security and development, degrading the quality of life and heavily impacting the economy with longer hospital stays, higher medical expenses and higher mortality. This is a natural phenomenon whose process is accelerated by the excessive use or misuse of these drugs in humans and animals. An increasing number of infections, such as pneumonia, tuberculosis or salmonellosis, are becoming more and more difficult to treat because of the lack of effectiveness of the antibiotics used. To circumvent this resistance, one of the tracks consists in developing new antibiotics with new biological targets. Tiacumicin B (Tcn-B) meets these criteria and, received marketing authorization in 2011 in the United States for the treatment of Clostridium difficile intestinal infections, which are often of nosocomial origin and had previously been fatal in $25 \%$ of the cases. ${ }^{1}$ Tcn-B interacts with bacterial RNA polymerase (RNAP) ${ }^{2}$ blocking RNA synthesis, a strategy already used in broad-spectrum antibacterial therapy. Since Tcn-B inhibits bacterial RNAP by binding a site that does not overlap with other antibiotic binding sites, there is no known cross-resistance with the other antibiotics in use. There is no cross-resistance with rifamycin, ${ }^{3}$ so Tcn-B is active on resistant strains of mycobacterium tuberculosis, which opens up new therapeutic possibilities. ${ }^{4}$ In this context, designing reliable total syntheses of Tcn-B that could also lead to analogues proves particularly relevant.

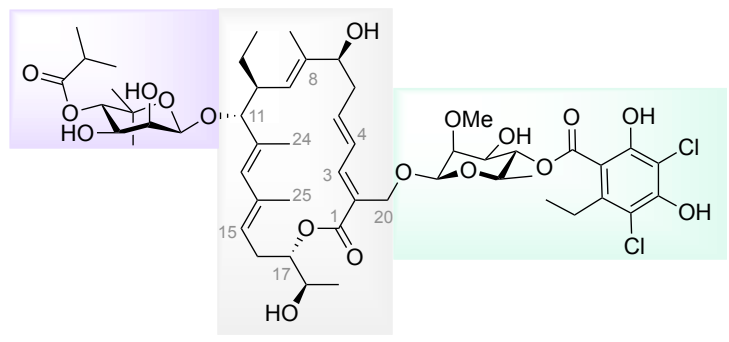

Figure 1. Tiacumicin B (Tcn-B)

Tcn-B is representative of a new class of antibiotic macrolides and is also known as clostomicin B1, fidaxomicin or lipiarmycin A3 (Figure 1). ${ }^{5}$ This natural product was first isolated in the 70 's from an actinobacterium, Actinoplanes deccanensis, found in the soil in India. ${ }^{6}$ Its 
structure was partially elucidated between 1983 and 1987 by Martinelli, ${ }^{7}$ Arnone and Nasini ${ }^{8}$ and recently its biosynthesis could be resolved by the team of Zhang. ${ }^{9}$ Tcn-B is among the most complex and heavier macrolide and its structure can be divided into three main parts: 1$)$ a central core composed of an eighteen-membered macrolactone displaying two $(E),(E)$-conjugated dienes, a trisubstituted $(E)$-alkene and featuring five stereogenic centers; 2) an eastern part with 2-O-methyl-D-rhamnose, linked as the $\beta$-anomer and esterified at the 4-position by an homodichloro-orsellinic acid; and 3) a western part consisting in a rare sugar, D-noviose, also linked as the $\beta$-anomer and esterified at the 4-position by isobutyric acid.

In 2015, aglycone's syntheses of Tcn-B were achieved by the Gadmann ${ }^{10}$ and Altmann ${ }^{11}$ groups while the Zhu ${ }^{12}$ group synthesized the macrolactonic core of a diastereomer of Tcn-B (OH on the C-18). In 2017, we also reported our own syntheses of the aglycone by designing two closely related pathways. ${ }^{13}$ The development of our strategy led us to discover a Kumada-Corriu reaction of vinyl sulfides catalyzed by Pd-nanoparticles. ${ }^{14} \mathrm{We}$ also reexplored the Grigg's allene/alkyne cross-coupling and proposed an unprecedented mechanism. ${ }^{15}$ Until 2020 , only the Gademann's team managed to complete the total synthesis of Tcn-B, providing solutions to the challenging problem of the 1,2-cisglycosylations but there was still room for innovation and improvement of selectivity. ${ }^{16}$ For the noviosylation step, the cyclic aglycone was found to show low reactivity toward a variety of different glycosyl donors or led exclusively to the $\alpha$-glycosylated adduct. The mercury(II) Helferich's protoco ${ }^{17}$ was then used for the glycosylation of an acyclic triene fragment with a noviosyl bromide giving the corresponding product in $63 \%$ yield $(\alpha / \beta: 1 / 3)$. After assembly and cyclization of the aglycone, the rhamnosylation was carried out on the macrolide with an acetimidate glycosyl donor delivering the fully protected Tcn-B $(\alpha / \beta$ : $1 / 4,62 \%)$. Note that recently De Brabander used similar glycosylation strategies for the synthesis of the northern and southern glycosylated fragments of Tcn-B. ${ }^{18}$ The total syntheses of tiacumicin $\mathrm{A}^{19}$, mangrolide $\mathrm{A}^{20}$ and $\mathrm{D},{ }^{21}$ three congeners of tiacumicin $\mathrm{B}$ with a slightly simplified aglycone structure were also achieved.

In this article we wish to report with more details on our total synthesis of Tcn-B, ${ }^{22}$ highlighting the original glycochemistry that we had to develop to achieve this goal. Displaying axial C-O bonds at C2, D-rhamnose and D-noviose can both be related to D-mannose derivatives, in which the methylhydroxyl group in the C5-position has been replaced by a methyl or a gem-dimethyl group, respectively. For this series, the glycosylation reactions leading to 1,2-cis derivatives are particularly challenging since the $\alpha$-compound is favored for steric and thermodynamic (anomeric effect) reasons and its $\mathrm{C}-2$ configuration precludes the application of conventional neighboring group participation effects. $^{23}$

Our total synthesis of Tcn-B relies on our syntheses of the Tcn-B aglycone, ${ }^{13}$ that are based on original and selective assembly of the three main regions of the molecule. We had originally imagined to sequentially glycosylate the aglycone, but finally opted for two more convergent retrosynthetic plans. In these scenarios, Tcn-B was disconnected into fragments A1, B1, and $\mathbf{C}$ or into fragments $\mathbf{A 2}, \mathbf{B 2}$, and $\mathbf{C}$ (Figure 2 ). ${ }^{13}$ Connection of fragment A1 together with fragment B1 would first be achieved using ruthenium-catalyzed cross metathesis while a Suzuki coupling would allow the assembly of vinylbromide B2 with the boronic ester A2. In both cases, following these steps, a ring-size selective macrolactonization would deliver a monoglycosylated aglycone having an unprotected $\mathrm{OH}$ at $\mathrm{C}-11$ ready for the noviosylation step with fragment $\mathbf{C}$. Knowing that the macrolactone has been described as a reluctant glycosyl acceptor, this challenging late $\beta$-glycosylation carried a high risk of failure. ${ }^{11}$

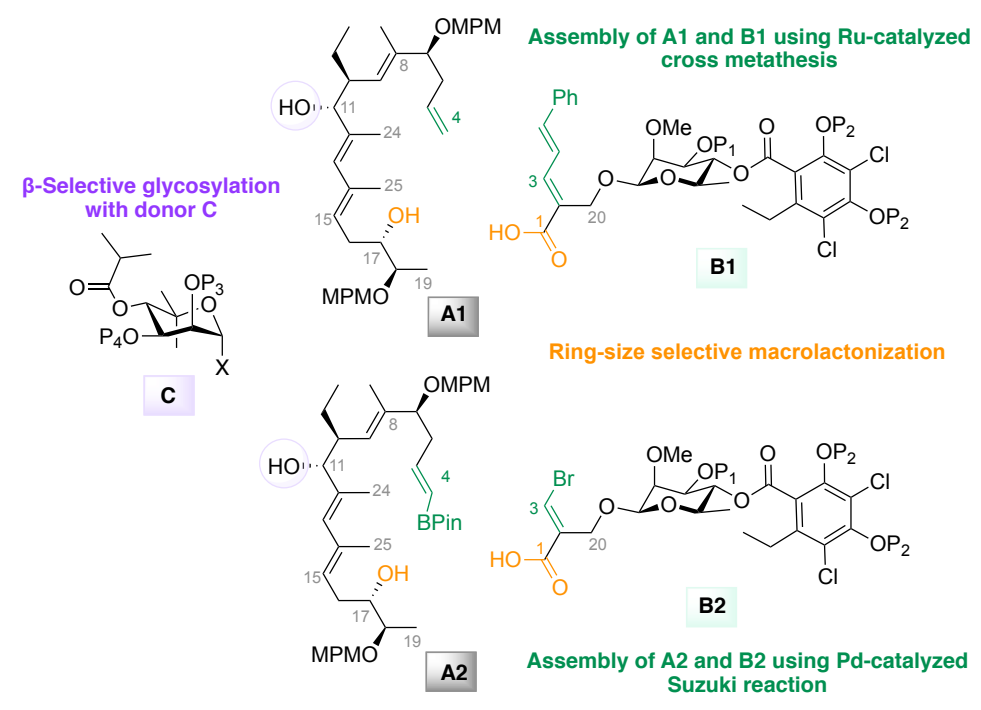

Figure 2. Pathways to tiacumicin B (Tcn-B).

\section{Preparation of the eastern part with 2-O-methyl-D-rhamnose.}

For the preparation of the rhamnose fragments B1 or B2, we initially considered using the mannose $\beta$-glycosylation method described by Kahne $^{24}$ and Crich. ${ }^{25}$ The activation of a mannosyl donor having a 4,6-benzylidene and an anomeric sulfoxide or a thioaryl leaving group 
would allow the selective formation of the $\beta$-mannoside compound through $\mathrm{S}_{\mathrm{N}} 2$ displacement of the corresponding $\alpha$-anomeric triflate. The obtained glycoside would therefore be later functionalized into a rhamnoside derivative.

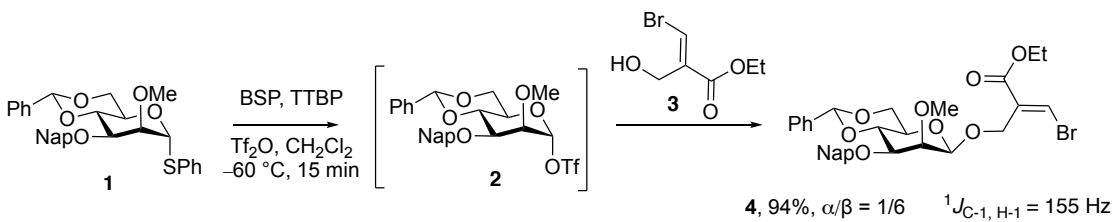

Scheme 1. Synthesis of $\beta$-mannoside 4. BSP: 1-benzenesulfinyl piperidine, TTBP: 2,4,6-tri-tert-butylpyrimidine.

To this aim, thioglycosyl donor 1 was prepared ${ }^{26}$ and submitted to Crich glycosylation conditions using pre-activation at $-60{ }^{\circ} \mathrm{C}$ by 1 benzenesulfinyl piperidine (BSP) in the presence of 2,4,6-tri-tert-butylpyrimidine (TTBP) and $\mathrm{Tf}_{2} \mathrm{O}$ (Scheme 1). ${ }^{27}$ The intermediate triflate was then allowed to react with the acceptor $3^{13 b}$ leading to the desired glycosylation product in a good $94 \%$ yield and a good selectivity of $1 / 6$ in favor of the $\beta$-adduct. The stereochemistry was determined by measuring the $\mathrm{C}-1 / \mathrm{H}-1$ coupling constant $\left({ }^{1} J_{\mathrm{C}-1, \mathrm{H}-1} \approx 160 \mathrm{~Hz}\right.$ if $\mathrm{H}-1$ is axial and ${ }^{1} J_{\mathrm{C}-1, \mathrm{H}-1} \approx 170 \mathrm{~Hz}$ if $\mathrm{H}-1$ is equatorial) derived from its ${ }^{1} \mathrm{H}$-coupled heteronuclear single quantum correlation spectrum. Unfortunately, we did not succeed in further functionalizing this compound into the desired rhamnoside. We then turned to a glycosylation using the phenylthiorhamnosyl donor 12 comprising the homodichloroorsellinate ester at the 4-position and a picoloyl group (Pico) at $O$-3 (Scheme 3, Table 1)). This glycosylation strategy, developed by Demchenko, involves intermolecular H-bonding between the nitrogen of the picoloyl group and the acceptor, directing the selective facial attack on the glycosyl donor on the same side as the Pico group. ${ }^{28}$ We started with the synthesis of orsellinate derivative 7 needed for the esterification at the 4-position of $S$-Phenyl-2- $O$-methyl-1-thio- $\alpha$-Drhamnopyranoside $\mathbf{1 0} .^{22}$ This compound can be synthesized in a straightforward manner from commercially available carboxylic acid $\mathbf{5 a}$ (Scheme 2). After dichlorination in acetic acid ${ }^{29}$ and formation of the cyclic ester using acetone and trifluoracetic anhydride in trifluoroacetic acid, ${ }^{30}$ the resulting phenol was protected as a 2-naphthylmethylidene ether (Nap) 6 . This protecting group was chosen as it could be removed using the same conditions (DDQ) as for 4-methoxybenzyl (MPM) ethers, already present on the aglycone. Lithiation of the benzylic methyl group of 6 with LDA at $-78{ }^{\circ} \mathrm{C}$ followed by trapping the corresponding anion with methyl iodide allowed the formation of the targeted 7 . Unfortunately, this reaction proved unreproducible giving yields ranging from 53 to $10 \%$ on a larger scale. For this reason, we chose a longer but reliable sequence starting from commercially available ethyl orsellinate $\mathbf{5 b}$. The latter was chlorinated and both phenols protected as tertbutyldimethylsilyl ethers provided 8. ${ }^{22}$ With this compound, the sequence of the deprotonation of the benzylic methyl group/methylation proceeded in an almost quantitative yield to furnish reproducibly the homologated compound. After acidic hydrolysis with concentrated sulfuric acid to the deprotected carboxylic acid 9, treatment with acetone and triflic anhydride in trifluoroacetic acid furnished the cyclic ester. Note that no reaction took place using trifluoracetic anhydride instead of $\mathrm{Tf}_{2} \mathrm{O}$. The remaining free phenol was finally protected, supplying Nap ether 7.

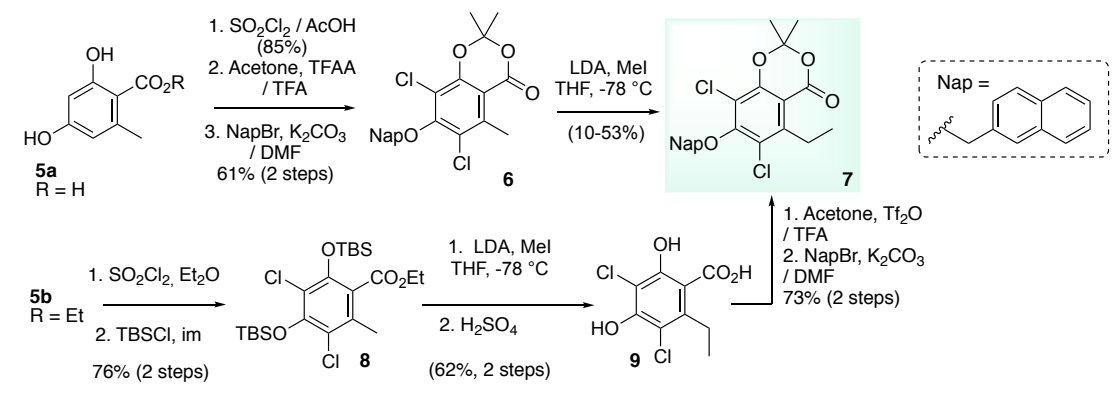

Scheme 2. Synthesis of orsellinate derivative 7. TFA: trifluoroacetic acid, TFAA: trifluoroacetic anhydride, NapBr: 2-naphthalene-methyl bromide, LDA: Lithium di-iso-propylamide

The synthesis of donor 12 was performed from diol 10 obtained in a few steps from phenyl-2,3,4,6-tetra- $O$-acetyl-1-thio- $\beta$-Dmannopyranoside (Scheme 3). ${ }^{22}$ Esterification of this diol using homodichloroorsellinate ester 7 and NaH in THF led to compound 11. As observed by Gademann et $a l .,{ }^{19}$ the $O-3$ position is first esterified and the desired adduct is formed after ester migration on a prolongated reaction time. The liberated phenol was then protected as 2-Nap ether and the $O-3$ position of the rhamnoside was esterified with picolinic acid to give the desired donor 12 . 


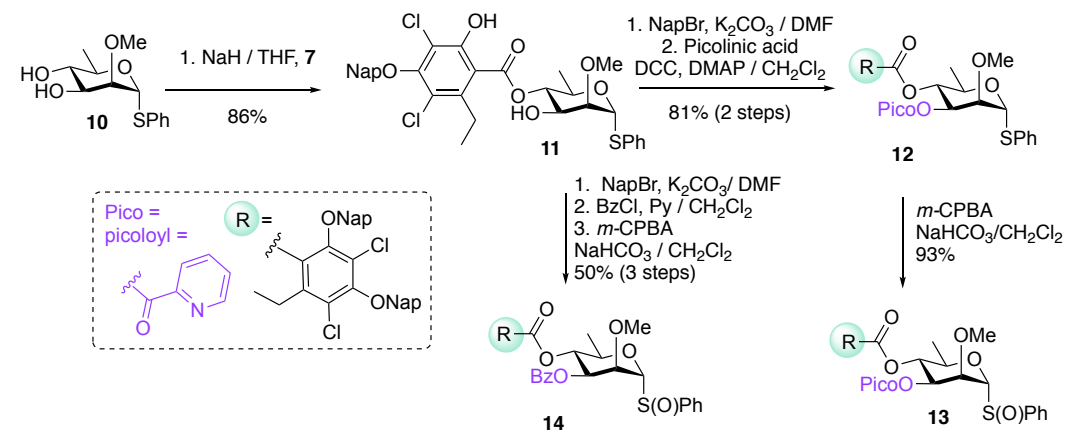

Scheme 3. Synthesis of rhamnopyranosyl donors 12-14. DCC: dicyclohexylcarbodiimide, DMAP: 4-dimethylaminopyridine, $m$-CPBA: 3Chloroperoxybenzoic acid.

Rhamnosyl donor 12 was first engaged in a glycosylation reaction with the monoprotected diol 16a as the acceptor (Table 1, entry 1). This compound was prepared from 2,2-dimethyl-1,3-dioxan-5-one ${ }^{31}$ after a Wittig reaction with bromotrimethylphenylphosphonium bromide in the presence of sodium bis(trimethylsilyl)amide (NaHMDS) in THF (Scheme 4). This was followed by deprotection of the acetonide and monoprotection of the resulting diol as TBS-ethers 15a and 15b. ${ }^{32}$ A Suzuki cross coupling of a mixture of 15a,b with phenylvinylboronic acid using catalytic $\mathrm{Pd}(\mathrm{OAc})_{2}$ combined with 2-dicyclohexylphosphino-2',6'-dimethoxybiphenyl (Sphos) as ligand and potassium phosphate in $\mathrm{THF} /$ water at room temperature was then carried out giving $\mathbf{1 6 a}$ and $\mathbf{1 6} \mathbf{b}$ that could be separated using preparative HPLC.

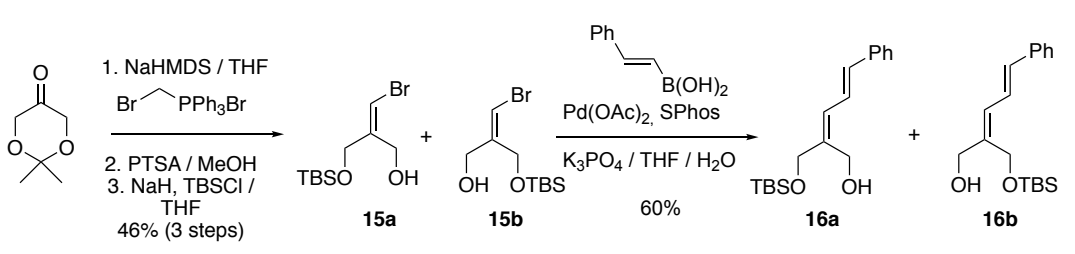

Scheme 4. Synthesis of acceptors 15a and 16a. NaHMDS: sodium bis(trimethylsilyl)amide, PTSA: para-toluene sulfonic acid, SPhos: 2dicyclohexylphosphino-2',6'-dimethoxybiphenyl.

The glycosylation reaction of 16a was carried out in 1,2-dichloroethane (DCE) with dimethyl(methylthio)sulfonium triflate (DMTST), a classical promoter used in H-bond-mediated Aglycone Delivery (HAD). However, no glycosylation adduct was obtained with donor 12, 16a proving very unstable under these conditions. Acceptor 15a was then used and with DMTST (2 eq.) in DCE at room temperature, we were pleased to obtain the glycosylation product 18 in $85 \%$ yield (Table 1, entry 2 ). A good $\alpha / \beta$ selectivity of $1 / 6.5$ was also achieved suggesting that HAD might have worked to mediate a $\beta$-selective glycosylation through an intermediate such as $\mathbf{2 1}$. Recrystallization allowed us to separate both anomers and to unambiguously determine the configuration of the major one through X-ray crystal diffraction analysis (Figure 3). ${ }^{33}$ The reaction was also carried out in dichloromethane using $N$-iodosuccinimide (NIS, 1.2 eq.) and a catalytic amount of triflic acid (TfOH, 0.24 eq.), which gave a good yield ( $86 \%)$ but with a slightly lower stereoselectivity $(\alpha / \beta=1: 4)$ (Table 1 , entry 3$)$.

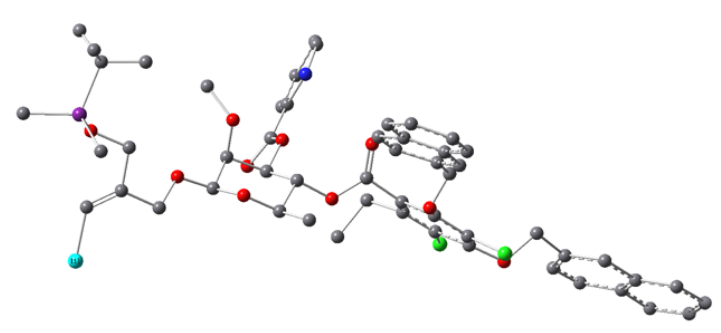

Figure 3. X-ray crystal structure of 18. 
Table 1. Rhamnosylation conditions of different acceptors with donors 12-14.

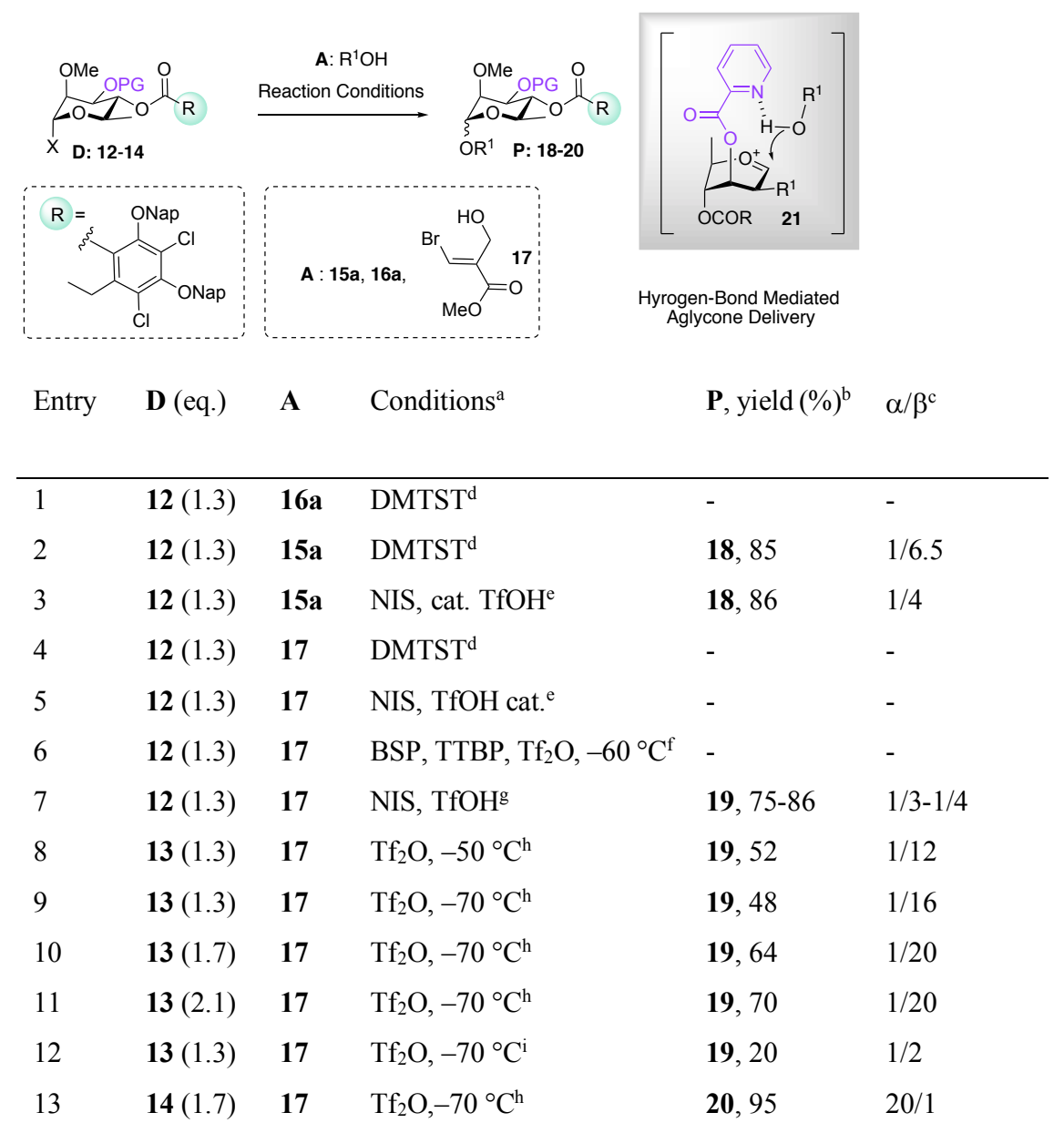

${ }^{a}$ Reaction performed in $\mathrm{CH}_{2} \mathrm{Cl}_{2}(c 0.01 \mathrm{M})$ with $4 \AA \mathrm{MS}$ unless otherwise stated. ${ }^{\mathrm{b}}$ After chromatography on silica gel. ${ }^{\mathrm{c}}$ Ratio and stereochemistry determined by ${ }^{1} \mathrm{H}$ NMR analysis of the crude mixture and by measuring ${ }^{1} J_{\mathrm{C} 1, \mathrm{H} 1}$ coupling respectively. ${ }^{\mathrm{d}}$ With 2.6 eq. of the promotor in DCE as solvent from $0{ }^{\circ} \mathrm{C}$ to r.t. ${ }^{\mathrm{e}}$. With 1.2 eq. of NIS and 0.24 eq. of TfOH from $-40{ }^{\circ} \mathrm{C}$ to r.t. ${ }^{\mathrm{f}}$ With 1.3 eq. of BSP, 2.5 eq. of TTBP and 1.4 eq. of $\mathrm{Tf}_{2} \mathrm{O} .{ }^{\mathrm{g}}$ With 1.2 eq. of NIS and 1 eq. of $\mathrm{TfOH}$ from $-40{ }^{\circ} \mathrm{C}$ to r.t. ${ }^{\mathrm{h}}$ With $1.3-1.9$ eq. of $\mathrm{Tf}_{2} \mathrm{O}, 3.5-4.6$ eq. of DTBMP and 4.2-6 eq. of ADMB. ${ }^{\text {i }}$ Preactivation protocol.
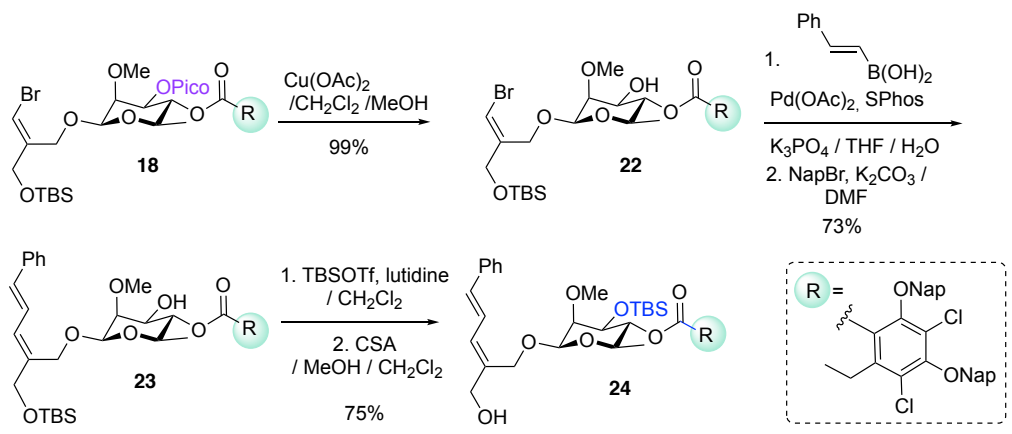

Scheme 5. Functionalization of 18. CSA: camphor sulfonic acid.

Before optimizing this glycosylation step, we chose to test the synthetic sequence leading to the key building block B1 necessitating a terminal carboxylic acid group. Thus, the Pico group in $\mathbf{1 8}$ was smoothly and selectively removed with $\mathrm{Cu}(\mathrm{OAc})_{2}$ in dichloromethane/methanol (Scheme 5). This was followed by a Pd-catalyzed Suzuki reaction with phenylvinylboronic acid using catalytic $\mathrm{Pd}(\mathrm{OAc})_{2} / \mathrm{Sphos}$ and $\mathrm{K}_{3} \mathrm{PO}_{4}$ in THF/water at room temperature for 18 hours. Following these mild conditions, we noticed the presence of 
about $20 \%$ of products displaying deprotected phenols on the orsellinate moiety. Direct treatment of the crude reaction mixture with NapBr in the presence of $\mathrm{K}_{2} \mathrm{CO}_{3}$ in DMF allowed us to reinstall the protective groups and obtain the targeted diene compound 23 in $73 \%$ yield. The $3-\mathrm{OH}$ on the rhamnoside moiety was then protected as a TBS-ether and the primary alcohol was deprotected using camphor sulfonic acid in methanol/ $\mathrm{CH}_{2} \mathrm{Cl}_{2}$, providing 24 in a 75\% yield. Unfortunately, we did not succeed in oxidizing this compound into the corresponding carboxylic acid prior to the next Ru-catalyzed cross metathesis with fragment $\mathbf{A 1}$.

We then turned our attention toward the glycosylation of the alternative acceptor $\mathbf{1 7}$ already bearing the carboxylic function. Contrary to previous results with 15a, activation of 12 with DMTST or NIS/cat. TfOH were disappointingly unsuccessful since no glycosylation adduct was observed (Table 1, entries 4 and 5). Activation with the $\mathrm{BSP} / \mathrm{Tf}_{2} \mathrm{O}$ method at $-60{ }^{\circ} \mathrm{C}$ in the presence of TTBP was also tried without success leading to the degradation of the acceptor (Table 1, entry 6). However, by increasing the amount of TfOH ( 0.92 eq./donor $)^{34}$ used in combination with $N$-iodosuccinimide ( 1 eq./donor) in $\mathrm{CH}_{2} \mathrm{Cl}_{2}$ at $-40{ }^{\circ} \mathrm{C}$ to r.t. produced 19 in a $76 \%$ yield as a mixture of anomers $(\alpha / \beta$ : 1/4) (Table 1, entry 7), that could be separated by preparative HPLC. Note that in this particular case, the excess of TfOH could disrupt the HAD pathway and the glycosylation would follow a different mechanism, probably involving the protonation of the nitrogen on the picoloyl and formation of a glycosyl triflate. ${ }^{35}$ By changing parameters such as temperature, promoter or donor amount, dilution, etc., we were not able to obtain a better selectivity. Seeking for higher $\beta$-selectivity, we decided to explore the reaction with sulfoxide 13, a type of anomeric leaving group never before used in combination with a directing picoloyl group. Following $m$-CPBA oxidation of sulfide 12, the activation of donor 13 (1.3 eq.) was first examined in $\mathrm{CH}_{2} \mathrm{Cl}_{2}$ at $-50{ }^{\circ} \mathrm{C}$ using $\mathrm{Tf}_{2} \mathrm{O}$ in the presence of the acceptor 17, 2,6-di-terbutyl-4-methylpiridine (DTBMP) as acid scavenger, and 4-allyl-1,2-dimethoxybenzene (ADMB) (Table 1, entry 8). ${ }^{36,37}$ We were pleased to find that under these conditions the desired glycosylated compound 19 with an upgraded facial selectivity $(\alpha / \beta: 1 / 12)$ and a yield of $52 \%$. Higher yield $(70 \%)$ and selectivity $(\alpha / \beta: 1 / 20)$ could be further attained by increasing the donor amount (2.1 eq.) and by lowering the temperature of the activation at $-70{ }^{\circ} \mathrm{C}$ (see Table 1, entries 8 to 11 for comparison). To verify the influence of the remote picoloyl group in stereodirecting the nucleophilic attack on the $\beta$-face by H-bonding, we carried out two control experiments. The first consisted in performing the reaction by pre-activating the donor using $\mathrm{Tf}_{2} \mathrm{O}$ at $-70{ }^{\circ} \mathrm{C}$ for 15 minutes followed by the addition of the acceptor (Table 1 , entry 12). These conditions led to the formation of the expected glycoside 19 in only $20 \%$ yield and poor selectivity $(\alpha / \beta: 1 / 2)$. The second control experiment was achieved with donor 14 having a benzoyl group in place of the picoloyl (Table 1, entry 13). This reaction led to pure $\alpha$-glycosylation product 20, indicating that the HAD mechanism may effectively take place only with the picoloyl group. The picoloyl of 19 was then removed with $\mathrm{Cu}(\mathrm{OAc})_{2}$ in $\mathrm{CH}_{2} \mathrm{Cl}_{2} / \mathrm{MeOH}$, and replaced by a TBS group to lead to fragment $\mathbf{B 2}$.

\section{Assemblage of A2 and B2 and macrolactonization}

We could easily scale up our synthesis of the aglycone fragment A2. The convergent step of Pd-catalyzed cross-coupling of boronic ester A2 with rhamnoside $\mathbf{B} 2$ proceeded cleanly with catalytic $\mathrm{Pd}(\mathrm{OAc})_{2} /$ Ruphos and potassium phosphate in a mixture of THF/water at r.t., furnishing ester $\mathbf{2 5}$ in 79\% yield (Scheme 6). As reported by Nicolaou et al., ${ }^{38}$ the use of $\mathrm{Me}_{3} \mathrm{SnOH}$ allowed us to hydrolyze selectively the methyl ester in the presence of the orsellinate on the rhamnoside moiety. Performed in toluene at $120^{\circ} \mathrm{C}$, the reaction led cleanly to secoacid in 70 to $93 \%$ yields. With the latter in hand, we then focused on a ring-size selective macrolactonization using Yamaguchi conditions as described for the synthesis of our aglycone. ${ }^{13}$ However, the transposition of these conditions on this substrate led to the desired hemiglycosylated tiacumicin B 26 in a low 23\% yield. With the Boden-Keck's protocol, ${ }^{39}$ the yield reached $58 \%$ yield, but $\mathbf{2 6}$ proved to be a mixture of two products resulting from the isomerization of the C4-C5 alkene. Finally, a far cleaner and reproducible macrolactonization was achieved using the Shiina's conditions ${ }^{40}$ with 2-methyl-6-nitrobenzoic anhydride furnishing 26 in $72 \%$ yield, and an isomerization minimized at $15 \%$. This selective strategy allowed us to keep the free $\mathrm{OH}$ at $\mathrm{C}-11$ so that $\mathbf{2 6}$ could be directly engaged in the next glycosylation step.

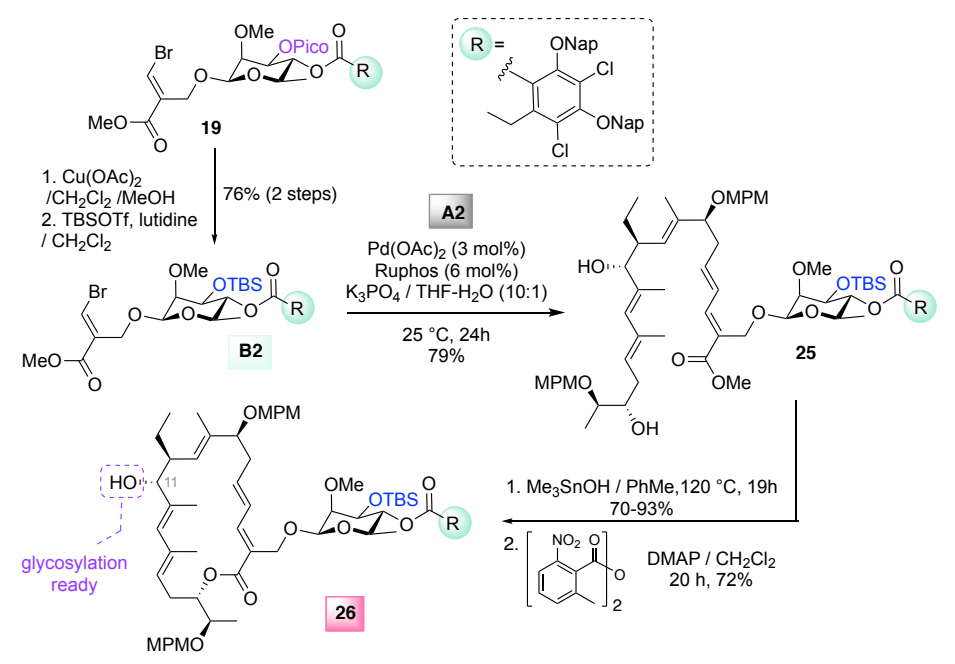


Scheme 6. Assemblage of $\mathbf{A 2}$ and $\mathbf{B 2}$ and macrolactonization to monoglycosylated 26. Ruphos : 2-dicyclohexylphosphino-2',6'diisopropoxybiphenyl.

\section{Preparation of the western part with D-niovose and noviosylation step.}

For the noviosylation, we had first programmed Intramolecular Aglycone Delivery (IAD) using a silicon tether anchoring $\mathbf{2 6}$ to the $2-O$ position of the noviosyl moiety. ${ }^{41}$ The axial configuration of the 2-hydroxyl group of the donor being favorable, this approach would $a$ priori provide a 1,2-cis glycosidic linkage with complete $\beta$-stereocontrol. For the preparation of the required noviosyl donor, we started from Darabinose (Scheme 7). Selective acetonide protection of the cis-diol, oxidation of the hemiacetal with trichloroisocyanuric acid (TCCA) and catalytic TEMPO followed by silylation of the remaining 2-OH group provided lactone 27. Introduction of the gem-dimethyl groups was achieved using the reaction of $\mathbf{2 7}$ with the methyl Grignard reagent and the corresponding diol was re-oxidized to lactone $\mathbf{2 8}$. Dibal-H reduction led to the lactol and a mild acidic treatment with formic acid allowed the selective removal of the acetonide protection, prior to the introduction of a thiophenyl group at the anomeric position. The resulting diol $\mathbf{2 9}$ was protected as dichloroacetyl esters, which upon treatment by $(i \mathrm{PrCO})_{2} \mathrm{O}$ in $\mathrm{MeCN}$ with a catalytic amount of $\mathrm{Sc}(\mathrm{OTf})_{3}$ allowed the direct replacement of the TBS group by an isobutyrate. ${ }^{42}$ The two dichloroacetates in the obtained compound were then selectively removed with sym-collidine in MeOH giving diol 30. The latter was then selectively mono-alkylated (NapBr, $\mathrm{CsF}$ ) after the prior formation of the stannylene $\mathbf{3 1}$ giving predominantly the compound $\mathbf{3 2}$ with 2-naphthylmethylidene ether at $O-2$ position $(2-O-\mathrm{Nap} / 3-O-\mathrm{Nap}=95 / 5)$ in $80 \%$ yield.
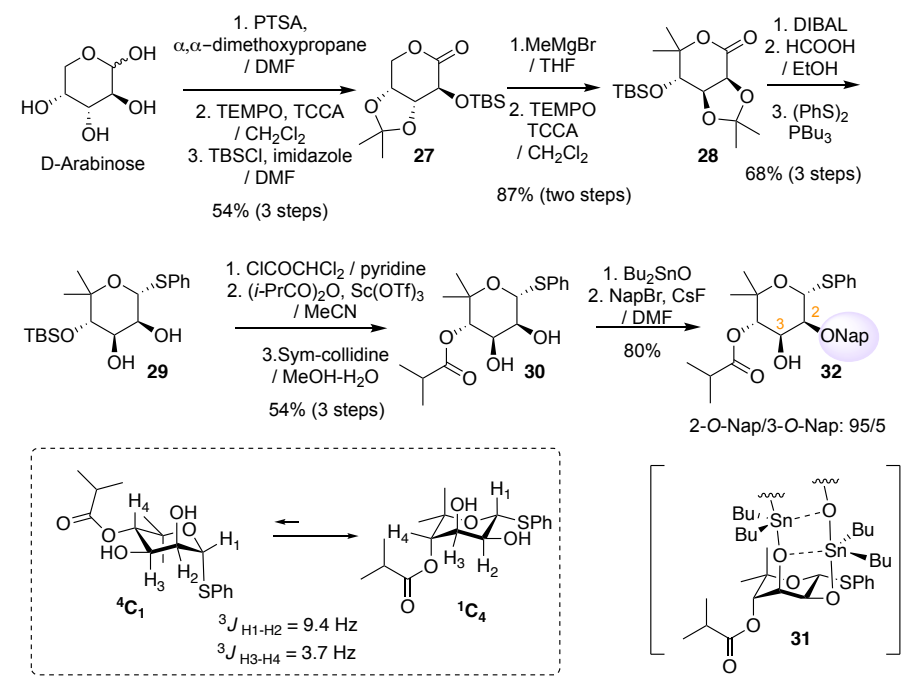

Scheme 7. Preparation of the noviosyl thioglycoside 32 from D-arabinose, conformational preference of diol $\mathbf{3 0}$ and likely structure of stannylene 31 with an equatorial C2-O2 bond. TEMPO: 2,2,6,6-tetramethyl-1-piperidinyloxy, TCCA: trichloroisocyanuric acid.

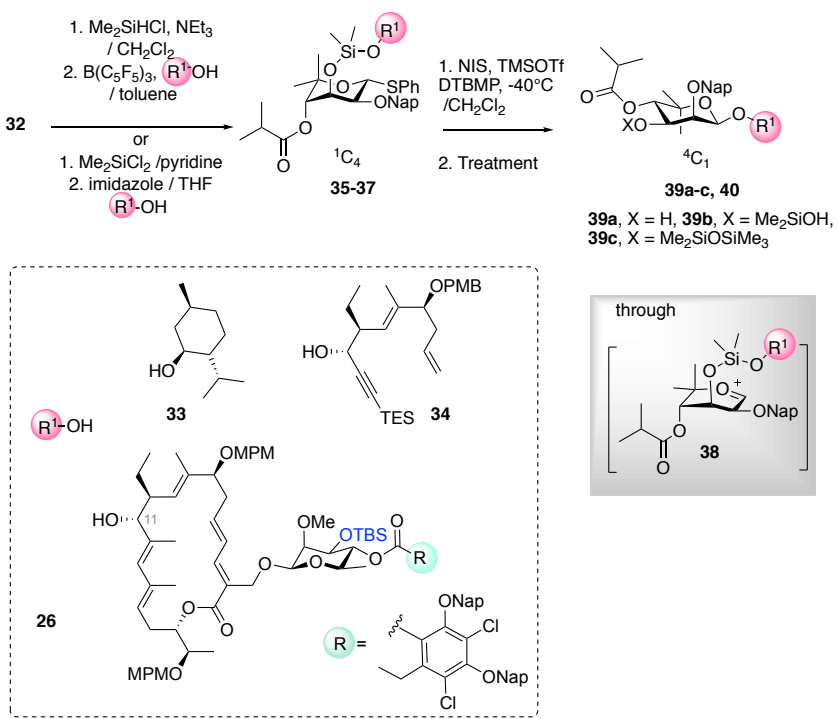

Scheme 8. Preparation of the silaketals 35-37 and glycosylation. NIS : N-iodosuccinimide; DTBMP: 2,6-Di-tert-butyl-4-methylpyridine. 
This unexpected selectivity can be rationalized by the preferred conformation of the starting diol 30, which is not the conventional ${ }^{4} \mathrm{C}_{1}$ chair but the ${ }^{1} \mathrm{C}_{4}$ chair, certainly imposed by the presence of the gem-dimethyl group. This was confirmed by the ${ }^{3} J_{1,2}$ coupling constant of $9.5 \mathrm{~Hz}$ in the ${ }^{1} \mathrm{H}$ NMR spectrum of diol 30 indicating a H-1/H-2 trans diaxial orientation. The equatorial $\mathrm{OH}$ bond being more accessible, this accounts for the selectivity of this protection.

We chose to investigate IAD approach with 32, although to our knowledge, no other example has been described with a glycosyl acceptor linked to the 3-O position of the donor (Scheme 8). Starting our study with (-)-menthol 33 as a model, the corresponding silaketal $\mathbf{3 5}$ could be easily synthesized with the recently reported method of Montgomery. ${ }^{41 \mathrm{c}}$ After treatment of noviosyl adduct 32 with dimethylchlorosilane, the corresponding alkoxysilane reacted with (-)-menthol 33 in the presence of $\mathrm{B}\left(\mathrm{C}_{6} \mathrm{~F}_{5}\right)_{3}$ as catalyst. As indicated by the ${ }^{1} \mathrm{H}$ NMR spectrum, the silylated compound $\mathbf{3 5}$ also adopts exclusively a ${ }^{1} \mathrm{C}_{4}$ chair conformation. Considering that intermediate oxonium 38, resulting from 35, must display a half-chair conformation (Scheme 8), the alcohol to be transferred must then occupy a position favorable for the formation of the desired $\beta$-derivative. Optimized glycosylation conditions on $\mathbf{3 5}$ were obtained when the reaction was carried out with NIS (1.3 eq.) and TMSOTf ( 1.8 eq.) in $\mathrm{CH}_{2} \mathrm{Cl}_{2}$ in the presence of DTBMP ( 3 eq.) at $-40{ }^{\circ} \mathrm{C}$ for $1 \mathrm{~h}$. Treatment of the reaction mixture with a solution of TBAF in THF led to the desired glycosylation product 39a $(\mathrm{X}=\mathrm{H})$ in $55 \%$ yield and as a single $\beta$-anomer. In this case, the obtained compound 39a adopted a ${ }^{4} \mathrm{C}_{1}$ conformation with a measured ${ }^{1} J_{\mathrm{C}-1, \mathrm{H}-1}$ of $159 \mathrm{~Hz}$ confirming the $\beta$-configuration. The moderate yield obtained here is likely due to the presence of the TBAF reagent that can cleave the $i$-butyrate ester. However, without TBAF treatment, we isolated, after silica gel chromatography, two stable glycosylation adducts bearing various silyl groups $\left(39 \mathbf{b}, \mathrm{X}=\mathrm{Me}_{2} \mathrm{SiOH}_{2}{ }^{43}\right.$ and $\left.39 \mathrm{c}, \mathrm{X}=\mathrm{Me}_{2} \mathrm{SiOTMS}{ }^{44}\right)$ at the 3$O$ position of the novioside. To avoid their formation, an anhydrous $\mathrm{HCl}$ solution in methanol was added after completion of the glycosylation which delivered targeted product 39a in a $81 \%$ yield with complete $\beta$-stereocontrol. The reaction was also carried out with the more complex alcohol 34, a synthetic precursor of the aglycone fragment A2. The formation of the silaketal was performed as with (-)-menthol which produced 36 exclusively in a ${ }^{1} \mathrm{C}_{4}$ chair conformation and a good $76 \%$ yield. Using the glycosylation conditions described previously and acid treatment, the corresponding adduct $\mathbf{4 0}$ was obtained in $41 \%$ yield also as a single $\beta$-anomer. As for 39, the obtained glycosylated compound 40 adopted a ${ }^{4} \mathrm{C}_{1}$ conformation with a measured ${ }^{1} J_{\mathrm{C}-1, \mathrm{H}-1}$ of $159 \mathrm{~Hz}$. Despite this unsatisfactory result, we decided to attempt the reaction with the semi-glycosylated tiacumicin B 26. In this case we failed at preparing the silaketal using Montgomery's conditions but good results were obtained with the preliminary formation of the chloroalkoxysilane ${ }^{41 a}$ that reacted with $\mathbf{2 6}$ providing the corresponding dialkoxysilane 37 in 73\% yield. Unfortunately, glycosylation using NIS and TMSOTf in DCM at $-40{ }^{\circ} \mathrm{C}$ followed by an $\mathrm{HCl}$ treatment led to degradation of the compound without evidence of the formation of the targeted glycosylated adduct.

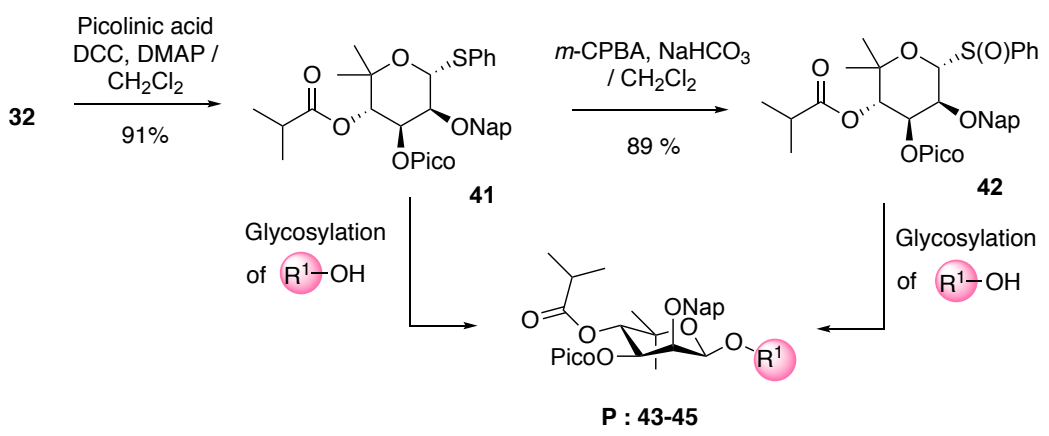

Scheme 9. Preparation of the noviosyl donors D (41-42) and glycosylation (structure of $\mathrm{R}^{1} \mathrm{OH}$ in Scheme 8).

We then shifted to another strategy based this time on an H-bond-mediated Aglycone Delivery (HAD) approach involving the use of noviosyl donor 41 bearing a Pico group at the 3-position (Scheme 9). Following esterification of $\mathbf{3 2}$ with picolinic acid, the corresponding sulfide $\mathbf{4 1}$ was engaged in a glycosylation reaction with (-)-menthol as the acceptor (Table 2, entry 1). The reaction was carried out with DMTST as the promoter and led predominantly to the $\beta$-compound $\mathbf{4 3}(\alpha / \beta: 1 / 5)$ in $83 \%$ yield. With elaborated unsaturated alcohol 34, this approach led to poor yields ( 11 to $21 \%$ ) either with DMTST (Table 2, entry 2) or NIS/TfOH (Table 2, entry 3 ) and unfortunately turned out unsuccessful with macrolactonic acceptor $\mathbf{2 6}$ since no glycosylated adduct was detected.

The success of the above-mentioned rhamnosylation led us to consider that sulfoxide $\mathbf{4 2}$ derived from sulfide $\mathbf{4 1}$ could be a far more reactive donor. A first trial with (-)-menthol as acceptor under $\mathrm{Tf}_{2} \mathrm{O}$ activation at $-70{ }^{\circ} \mathrm{C}$ revealed the potency of this method, as the $\beta$-anomer 43 was obtained as the only adduct in $66 \%$ yield (Table 2, entry 4). With alcohol 34, the use of the sulfoxide approach proved to be more efficient as well providing $\beta$-glycosylation product 44 in a $62 \%$ yield (Table 2, entry 5). Moreover, these reaction conditions applied to the hemi-glycosylated tiacumicin B $\mathbf{2 6}$ delightfully furnished the desired noviosylated product $\mathbf{4 5}$ in $68 \%$ yield, with high facial selectivity (Table 2, entry $6, \alpha / \beta>1 / 20$ ). 
Table 2. Glycosylation conditions of donors D (41-42) with different acceptors.

\begin{tabular}{|c|c|c|c|c|c|}
\hline Entry & $\mathbf{D}$ (eq.) & $\mathbf{R}^{1}-\mathbf{O H}$ & Conditions $^{\mathrm{a}}$ & $\mathbf{P}$, yield $(\%)^{\mathrm{b}}$ & $\alpha / \beta^{c}$ \\
\hline 1 & $41(1.3)$ & 33 & $\mathrm{DMTST}^{\mathrm{d}}$ & 43,83 & $1 / 5$ \\
\hline 2 & $41(1.3)$ & 34 & $\mathrm{DMTST}^{\mathrm{d}}$ & 44,11 & nd \\
\hline 3 & $41(1.3)$ & 34 & NIS, $\mathrm{TfOH}^{\mathrm{e}}$ & $\mathbf{4 4}, 21$ & nd \\
\hline 4 & $42(1.3)$ & 33 & $\mathrm{Tf}_{2} \mathrm{O},-70{ }^{\circ} \mathrm{C}^{\mathrm{f}}$ & 43,66 & $>1 / 20$ \\
\hline 5 & $42(2.1)$ & 34 & $\mathrm{Tf}_{2} \mathrm{O},-70{ }^{\circ} \mathrm{C}^{\mathrm{g}}$ & 44,62 & $1 / 20$ \\
\hline 6 & $42(2.1)$ & 26 & $\mathrm{Tf}_{2} \mathrm{O},-70^{\circ} \mathrm{C}^{\mathrm{g}}$ & 45,68 & $>1 / 20$ \\
\hline
\end{tabular}

${ }^{a}$ Reaction performed in $\mathrm{CH}_{2} \mathrm{Cl}_{2}(c 0.01 \mathrm{M})$ with $4 \AA \mathrm{MS}$ unless otherwise stated. ${ }^{\mathrm{b}}$ After chromatography on silica gel. ${ }^{\mathrm{c}}$ Ratio and stereochemistry determined by ${ }^{1} \mathrm{H}$ NMR analysis of the crude mixture and by measuring ${ }^{1} J_{\mathrm{C} 1, \mathrm{H} 1}$ coupling. ${ }^{\mathrm{d}}$ With 2.3 eq. of the promotor in DCE $(0.02 \mathrm{M})$ from $0{ }^{\circ} \mathrm{C}$ to r.t. ${ }^{\mathrm{e}}$ With 1.4 eq. of NIS and 1.8 eq. of TfOH from $-40{ }^{\circ} \mathrm{C}$ to r.t. ${ }^{\mathrm{f}}$ With 1.5 eq. of Tf $\mathrm{T}_{2} \mathrm{O}$ and 3 eq. of DTBMP. $\mathrm{g}$ With 1.9 eq. of $\mathrm{Tf}_{2} \mathrm{O}, 4.7$ eq. of DTBMP and 4.2 eq. of $\mathrm{ADMB}$.

\section{Final deprotection stages}

The last steps consisting in the removal of all protective groups (2 MPM, 3 Nap, 1 Pico and 1 TBS) from compound 45 proved unpleasantly more difficult than expected (Scheme 10). Using HF.NEt ${ }_{3}$ in THF allowed us to remove first the TBS group located on the rhamnosyl moiety giving the corresponding alcohol. The $2 \mathrm{MPM}$ as well as the Nap located on the novioside were then oxidized with $\mathrm{DDQ}$ in $\mathrm{CH}_{2} \mathrm{Cl}_{2} / \mathrm{H}_{2} \mathrm{O}$. At this stage, the two Nap groups protecting the phenol functions of the rhamnosyl moiety resisted these conditions at $0{ }^{\circ} \mathrm{C}$, and a longer reaction time at $20{ }^{\circ} \mathrm{C}$ led to an intractable mixture of products. The removal of the picoloyl was then cleanly carried out using $\mathrm{Cu}(\mathrm{OAc})_{2}$ in $\mathrm{CH}_{2} \mathrm{Cl}_{2} / \mathrm{MeOH}$ at $0{ }^{\circ} \mathrm{C}$ to produce 46. This deprotection sequence order was important as the Pico group had to be removed after its neighboring Nap group to avoid the DDQ-promoted formation of a 2,3-O-naphthylmethylidene on the novioside. These three operations were performed with no intermediate purifications providing an overall yield of $74 \%$ of $\mathbf{4 6}$. However, the unexpected problem of cleavage of the two Nap groups located on the two phenol functions remained to be addressed. Lewis acid-mediated treatment of the Nap led only to the degradation of the molecule. Pd-catalyzed hydrogenation $(\mathrm{Pd} / \mathrm{C}$, cyclohexene) allowed the phenol deprotection but along with the reduction of the C4-C5 alkene. ${ }^{45}$ As above mentioned, we observed a partial loss of the Nap groups located on the phenol moiety during the Pd-catalyzed Suzuki reaction of $\mathbf{2 3}$ with phenyl vinylboronic acid (see Scheme 5 and text). Exploiting this observation, we finally discover a new and selective method of deprotection of Nap ether on phenol. After a few optimizations, we found that using $\mathrm{Pd}_{2}(\mathrm{dba})_{3}$ as a catalyst in combination with $4 \mathrm{PPh}_{3}$ along with 1,3-dimethylbarbituric acid (NDMBA) as methyl naphthyl scavenger and pyridine in DMF at $80{ }^{\circ} \mathrm{C}$ provided selective and smooth deprotection conditions. This ultimate step supplied the target compound tiacumicin B in $73 \%$ yield, with chemical data identical to those of the naturally occurring compound. ${ }^{6 b}$

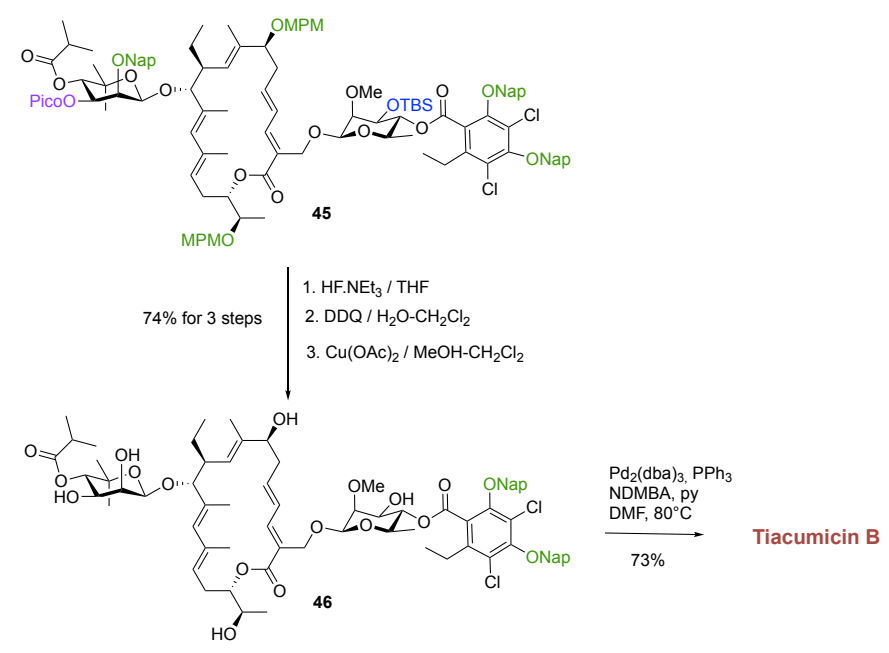


In conclusion we have achieved a convergent total synthesis of tiacumicin B, by assembling the three main regions of the molecule. A highly $\beta$-selective rhamnosylation of the C1-C3 fragment followed by a Suzuki cross-coupling allowed assembling the rhamnoside 19 with the C4C19 aglycone fragment A2. A ring-size selective macrolactonization using Shiina conditions was carried out followed by a final highly selective $\beta$-noviosylation of the cyclic aglycone and removal of all the protecting groups. During our glycosylation studies, we discovered a novel variant of the Demchenko procedure thanks to the conjoint use of a phenylsulfoxide leaving-group and a remote 3-O-picoloyl group on the donor. This combination allowed us to solve the problem of 1,2-cis glycosylation with a sensitive and complex aglycone and to reach a remarkable facial selectivity relying on an $\mathrm{H}$-bond-directed effect. This new procedure will certainly prove useful in addressing the biological relevance of the tiacumicin B carbohydrate sugars or for the preparation of a set of glycosylated analogues. We also found a new and effective method for the removal of a 2-naphthylmethylidene (Nap) ether protecting group on a phenol through the use of palladium catalysis.

\section{ASSOCIATED CONTENT}

\section{Supporting Information}

Crystallographic data for structure 18, preparation of all of the compounds, ${ }^{1} \mathrm{H}$ and ${ }^{13} \mathrm{C}$ NMR spectra for new compounds $\mathbf{4 , 6}, \mathbf{1 5}-\mathbf{1 6}, \mathbf{1 8}, \mathbf{2 2}-$ 24, 39-40, 43-44, ${ }^{1} \mathrm{H}$ and ${ }^{13} \mathrm{C}$ NMR spectra of the synthetic and natural Ten-B

\section{ACKNOWLEDGMENT}

We gratefully acknowledge financial supports from the French Agence Nationale pour la Recherche (ANR-14-CE16-0019-02, SYNTIA project), the CNRS, and the Université de Paris. We thank Karim Hammad and Jean-François Gallard for NMR support and Rosemary Green Beau for her editing of the manuscript.

\section{CONFLICT OF INTEREST}

The authors declare no conflict of interest.

\section{REFERENCES}

\footnotetext{
${ }^{1}$ Traynor, K., Am. J. Health Syst. Pharm. 2011, 68 (14), 1276-1276.

2 Tupin, A.; Gualtieri, M.; Leonetti, J.-P.; Brodolin, K., The EMBO J. 2010, 29 (15), 2527-2537. b) Gualtieri, M.; Tupin, A.; Brodolin, K.; Leonetti, J.-P., Int. J. Antimicrobial Agents 2009, 34 (6), 605-606.

${ }^{3}$ Srivastava, A.; Talaue, M.; Liu, S.; Degen, D.; Ebright, R. Y.; Sineva, E.; Chakraborty, A.; Druzhinin, S. Y.; Chatterjee, S.; Mukhopadhyay, J.; Ebright, Y. W.; Zozula, A.; Shen, J.; Sengupta, S.; Niedfeldt, R. R.; Xin, C.; Kaneko, T.; Irschik, H.; Jansen, R.; Donadio, S.; Connell, N.; Ebright, R. H., Curr. Opin. Microbiol. 2011, 14 (5), 532-543.

${ }^{4}$ Kurabachew, M.; Lu, S. H. J.; Krastel, P.; Schmitt, E. K.; Suresh, B. L.; Goh, A.; Knox, J. E.; Ma, N. L.; Jiricek, J.; Beer, D.; Cynamon, M.; Petersen, F.; Dartois, V.; Keller, T.; Dick, T.; Sambandamurthy, V. K., J. Antimicrob. Chemother. 2008, 62 (4), 713-719.

${ }^{5}$ For recent reviews on chemistry and biology of tiacumicin B see : a) Roulland, E., Synthesis 2018, 50 (21), 4189-4200. b) Dorst, A.;

Gademann, K., Helv. Chim. Acta 2020, 103 (4), e2000038.

${ }^{6}$ a) Parenti, F.; Pagani, H.; Beretta, G., J. Antibiot. 1975, 28 (4), 247-252. b) Coronelli, C.; White, R. J.; Lancini, G. C.; Parenti, F., J. Antibiot. 1975, 28 (4), 253-259.

${ }^{7}$ Martinelli, E.; Faniuolo, L.; Tuan, G.; Gallo, G. G.; Cavalleri, B., J. Antibiot. 1983, 36 (10), 1312-1322.

${ }^{8}$ Arnone, A.; Nasini, G.; Cavalleri, B., J. Chem. Soc., Perkin Trans. 1 1987, 1353-1359.

${ }^{9}$ a) Xiao, Y.; Li, S.; Niu, S.; Ma, L.; Zhang, G.; Zhang, H.; Zhang, G.; Ju, J.; Zhang, C., J. Am. Chem. Soc. 2011, 133 (4), 1092-1105. b)

Yu, Z.; Zhang, H.; Yuan, C.; Zhang, Q.; Khan, I.; Zhu, Y.; Zhang, C., Org. Lett. 2019, 21 (18), 7679-7683.

${ }^{10}$ Miyatake-Ondozabal, H.; Kaufmann, E.; Gademann, K., Angew. Chem., Int. Ed. 2015, 54 (6), 1933-1936.

${ }^{11}$ Glaus, F.; Altmann, K. H., Angew. Chem., Int. Ed. 2015, 54 (6), 1937-1940.

${ }^{12}$ Erb, W.; Grassot, J. M.; Linder, D.; Neuville, L.; Zhu, J., Angew. Chem., Int. Ed. 2015, 54 (6), 1929-1932.

13 a) Jeanne-Julien, L.; Masson, G.; Astier, E.; Genta-Jouve, G.; Servajean, V.; Beau, J.-M.; Norsikian, S.; Roulland, E., Org. Lett. 2017, 19 (15), 4006-4009. b) Jeanne-Julien, L.; Masson, G.; Astier, E.; Genta-Jouve, G.; Servajean, V.; Beau, J. M.; Norsikian, S.; Roulland, E., J. Org. Chem. 2018, 83 (2), 921-929.

14 Jeanne-Julien, L.; Astier, E.; Lai-Kuen, R.; Genta-Jouve, G.; Roulland, E., Org. Lett. 2018, 20 (5), 1430-1434.

15 Jeanne-Julien, L.; Masson, G.; Kouoi, R.; Regazzetti, A.; Genta-Jouve, G.; Gandon, V.; Roulland, E., Org. Lett. 2019, 21 (9), $3136-$ 3141.

${ }^{16}$ Kaufmann, E.; Hattori, H.; Miyatake-Ondozabal, H.; Gademann, K., Org Lett 2015, 17, 3514-3517.

17 Helferich, B.; Wedemeyer, K. F., Liebigs Ann. Chem. 1949, 563, 139-145.

${ }^{18}$ Hollibaugh, R.; Yu, X.; De Brabander, J. K., Tetrahedron 2020. DOI : 10.1016/j.tet.2020.131673

${ }^{19}$ Hattori, H.; Kaufmann, E.; Miyatake-Ondozabal, H.; Berg, R.; Gademann, K., J. Org. Chem. 2018, 83 (13), $7180-7205$.
} 
${ }^{20}$ Hattori, H.; Roesslein, J.; Caspers, P.; Zerbe, K.; Miyatake-Ondozabal, H.; Ritz, D.; Rueedi, G.; Gademann, K., Angew. Chem., Int. Ed. 2018, 57 (34), 11020-11024.

${ }^{21}$ Hattori, H.; Hoff, L. V.; Gademann, K., Org. Lett. 2019, 21 (9), 3456-3459.

22 a) Norsikian, S.; Tresse, C.; François-Eude, M.; Jeanne-Julien, L.; Masson, G.; Servajean, V.; Genta-Jouve, G.; Beau, J. M.; Roulland, E., Angew. Chem, Int. Ed. 2020, 59 (16), 6612-6616. b) Norsikian, S.; Tresse, C.; François-Eude, M.; Jeanne-Julien, L.; Masson, G.; Servajean, V.; Genta-Jouve, G.; Beau, J.-M.; Roulland, E., Angew. Chem. 2020, 132 (16), 6674-6678.

${ }^{23}$ For a recent review see: Nigudkar, S. S.; Demchenko, A. V., Chem. Sci. 2015, 6 (5), 2687-2704.

${ }^{24}$ Kahne, D.; Walker, S.; Cheng, Y.; Van Engen, D., J. Am. Chem. Soc. 1989, 111, 6881.

${ }^{25}$ Crich, D.; Sun, S., J. Org. Chem. 1996, 61 (14), 4506-4507.

${ }^{26}$ Elferink, H.; Mensink, R. A.; Castelijns, W. W. A.; Jansen, O.; Bruekers, J. P. J.; Martens, J.; Oomens, J.; Rijs, A. M.; Boltje, T. J., Angew. Chem., Int. Ed. 2019, 58 (26), 8746-8751

${ }^{27}$ Crich, D.; Smith, M., J. Am. Chem. Soc. 2001, 123 (37), 9015-9020.

28 a) Yasomanee, J. P.; Demchenko, A. V., J. Am. Chem. Soc. 2012, 134 (49), 20097-20102. b) Pistorio, S. G.; Yasomanee, J. P.; Demchenko, A. V., Org. Lett. 2014, 16 (3), 716-719.

${ }^{29}$ Milligan, R. F.; Hope, F. J., J. Am. Chem. Soc. 1941, 63 (2), 544-544.

${ }^{30}$ Marriott, J. H.; Barber, A. M. M.; Hardcastle, I. R.; Rowlands, M. G.; Grimshaw, R. M.; Neidle, S.; Jarman, M., J. Chem. Soc., Perkin Trans. 1 2000, (24), 4265-4278.

${ }^{31}$ González-García, E. M.; Grognux, J.; Wahler, D.; Reymond, J.-L., Helv. Chim. Acta 2003, 86 (7), 2458-2470.

${ }^{32}$ As the reaction is completely unselective, both isomers were separated on preparative HPIC. The stereochemistry was determined by NMR spectroscopy using NOESY experiments.

${ }^{33}$ See the Supporting Information. CCDC-2027426 contains the supplementary crystallographic data for this paper. These data can be obtained free of charge from The Cambridge Crystallographic Data Centre via www.ccdc.cam.ac.uk/ data_request/cif.

${ }^{34}$ a) Escopy, S.; Geringer, S. A.; De Meo, C., Org. Lett. 2017, 19 (10), 2638-2641; b) Mannino, M. P.; Demchenko, A. V., Chem. Eur. J. 2020, 26 (13), 2938-2946.

${ }^{35}$ Mannino, M. P.; Demchenko, A. V., Chem. Eur. J. 2020, 26 (13), 2927-2937.

${ }^{36}$ ADMB scavenge phenylsulfenyl triflate, a highly reactive byproduct, which forms after activation of anomeric sulfoxides with $\mathrm{Tf}_{2} \mathrm{O}$. Gildersleeve, J.; Smith, A.; Sakurai, K.; Raghavan, S.; Kahne, D., J. Am. Chem. Soc. 1999, 121 (26), 6176-6182.

${ }^{37}$ Zeng, J.; Liu, Y.; Chen, W.; Zhao, X.; Meng, L.; Wan, Q., Top Curr Chem (Cham) 2018, 376 (4), 27.

${ }^{38}$ Nicolaou, K. C.; Estrada, A. A.; Zak, M.; Lee, S. H.; Safina, B. S., Angew. Chem., Int. Ed. Eng.l 2005, 44 (9), $1378-1382$.

${ }^{39}$ Boden, E. P.; Keck, G. E., J. Org. Chem. 1985, 50 (13), 2394-2395.

${ }^{40}$ Shiina, I.; Kubota, M.; Oshiumi, H.; Hashizume, M., J. Org. Chem. 2004, 69 (6), 1822-1830.

${ }^{41}$ a) Stork, G.; Kim, G., J. Am. Chem. Soc. 1992, 114, 1087. Bols, M., J. Chem. Soc., Chem. Commun. 1992, (12), 913-914. c) Walk, J. T.; Buchan, Z. A.; Montgomery, J., Chem. Sci. 2015, 6 (6), 3448-3453.

${ }^{42}$ Norsikian, S.; Holmes, I.; Lagasse, F.; Kagan, H. B., Tetrahedron Lett. 2002, 43 (33), 5715-5717.

${ }^{43}$ Packard, G. K.; Rychnovsky, S. D., Org. Lett. 2001, 3 (21), 3393-3396.

${ }^{44}$ Beignet, J.; Cox, L. R., Org. Lett. 2003, 5 (22), 4231-4234.

${ }^{45}$ Smith, III, A B.; Sfouggatakis, C.; Risatti, C. A.; Sperry, J. B.; Zhu, W.; Doughty, V. A.; Tomioka, T.; Gotchev, D. B.; Bennett, C. S.; Sakamoto, S.; Atasoylu, O.; Shirakami, S.; Bauer, D.; Takeuchi, M.; Koyanagi, J.; Sakamoto, Y. Tetrahedron 2009, 65, $6489-6509$. 